\title{
Zur Kenntnis der Extraktivstoffe der Muskeln.
}

\author{
XIII. Mitteilung.
}

Über die Fällbarkeit einiger stickstoffhaltigen Extraktivstoffe durch Phosphorwolframsäure und Quecksilberoxydsalze.

\section{Von}

S. Demjanowski, stud. med.

(Aus dem medizinisch-chemischen Laboratorium der Universität Moskau.

(Der Redaktion zugegangen am 8. Juli 1912.)

Da es in der Literatur an Angaben über den Empfindlichkeitsgrad der Quecksilberoxydsalze als Reagenzien zur Fällung des Kreatinins fast ganz fehlt und keine genauen Hinweise auf die Fällbarkeit des Carnosins und des Methylguanidins durch Phosphorwolframsäure und Quecksilberoxydsalze vorhanden sind, so unternahm ich auf Herrn Prof. Dr. Wl. Gulewitschs Vorschlag hin und unter seiner unmittelbaren Leitung die Erforschung dieser Reaktionen.

Als Fällungsmittel benutzte ich vier Quecksilberoxydsalze (Sublimat, das essigsaure, schwefelsaure und salpetersaure Quecksilberoxyd) und Phosphorwolframsäure. Die Lösungen dieser Reagenzien wurden folgendermaßen bereitet:

Sublimat, eine bei Zimmertemperatur gesättigte wässerige Lösung.

Quecksilberoxydacetat, eine wässerige Lösung.

Quecksilberoxydsulfat, eine Lösung von $25 \mathrm{~g}$ Salz in $250 \mathrm{ccm}$ Wasser; das Wasser wurde zum Quecksilberoxydsulfat in kleinen Portionen zugegeben und das ausgefallene basische Quecksilbersalz abfiltriert.

Quecksilberoxydsulfat in 5\% 0 iger Schwefelsäure, die Lösung wurde wie die vorige, nur mit dem Unterschied hergestellt, daß anstatt Wasser 5\% ige Schwefelsäure benutzt wurde.

Quecksilberoxydnitrat, die wässerige Lösung wurde ebenso wie die von Quecksilberoxydsulfat bereitet. 
Phosphorwolframsäure (PwS), eine 2,5\% ige und 25\%ige wässerige Lösung der nach Drechsel1) bereiteten Säure.

Als Versuchssubstanzen wurden drei Verbindungen genommen:

Freies Kreatinin, welches ich nach Folins Verfahren dargestellt habe; die Substanz wurde aus Alkohol umkrystallisiert und im Vakuumexsikkator getrocknet.

0,1292 g Substanz gaben 41,55 ccm Stickstoff bei $17^{\circ}$ und $761,5 \mathrm{~mm}$ Bar.

$$
\begin{array}{cc}
\text { Gefunden: } & \text { Berechnet für } \mathrm{C}_{4} \mathrm{H}_{7} \mathrm{~N}_{3} \mathrm{O}: \\
\mathrm{N}=37,11 \% & 37,17 \% .
\end{array}
$$

Freies Carnosin, von Prof. Dr. Wl. Gulewitsch aus Liebigschem Fleischextrakt gewonnen; $[\alpha]_{546}^{20}=+25,0^{\circ}$ bei $c=13,004 \%$. Für die Überlassung eines Teiles dieses Präparates zu meiner Untersuchung spreche ich Herrn Prof. Dr. Wl. Gulewitsch auch an dieser Stelle meinen Dank aus.

Methylguanidinnitrat, von mir aus Kreatin durch Oxydation mittels Quecksilberoxyd erhalten. Die Identität dieses Präparates mit Methylguanidinnitrat wurde durch die Bestimmung des Schmelzpunktes $\left(150^{\circ}\right)$ und die von Herrn Prof. Dr. WI. Gulewitsch in liebenswürdiger Weise ausgeführte krystallographische Untersuchung festgestellt.

Zum Fällen nahm ich immer je $5 \mathrm{ccm}$ der Lösung der zu untersuchenden Substanz und gab das Reagens tropfenweise zu. Bei der Prüfung der Löslichkeit der Niederschläge dekantierte ich zuerst die sämtliche Flüssigkeit und gab dann das Reagens zu.

Die Resultate meiner Untersuchnngen führten mich $\mathrm{zu}$ folgenden Schlüssen.

Für das Kreatinin erwies sich als bestes Fällungsmittel die Phosphorwolframsäure, sowohl die 2,5\% ige als die 25\% ige. Beide Lösungen bewirken eine Fällung bei einer Verdünnung der Substanz von 1:25000, wobei mit der 2,5\% igen Säurelösung der Niederschlag sich erst nach längerem Stehen bildet: 1909, S. 787.

1) F. Hoppe-Seyler's Handb. d. path. u. phys.-chem. Analyse, 
Nach Fr. Hofmeisters ${ }^{1}$ ) Beobachtungen gibt eine Lösung Kreatinin 1:12000 mit Phosphorwolframsäure nach 24 Stunden einen krystallinischen Niederschlag. Sublimat gibt nach meiner Erfahrung eine Fällung mit Kreatininlösungen von ungefähr derselben Verdünnung $(1: 3000)$, wie bei Fr. Hofmeister (1. c.) angegeben ist (weniger als $1: 2000$ ). Die übrigen Quecksilberoxydsalze bewirken keine Fällung, selbst bei 1\% Kreatiningehalt in der Lösung.

Carnosin wird sehr gut von Quecksilberoxydsulfat in $5 \%$ iger Schwefelsäure und Quecksilberoxydnitrat gefällt, wobei bei der Anwendung des ersten dieser Reagenzien die Fällung rascher vor sich geht. Sublimat bewirkt eine Fällung bei einer Verdünnung von 1:2000, doch nur bei längerem Stehen ( 8 bis $10 \mathrm{St}$.). 2,5\% \% ige Phosphorwolframsäure fällt Carnosin bei einer Verdünnung von 1:9000, wobei der Niederschlag nach 24 Stunden entsteht; in demselben Zeitraum wird es von 25\% iger PwS in einer Lösung 1:20000 gefällt.

Methylguanidinnitrat gibt mit Quecksilberoxydacetat und Quecksilberoxydnitrat einen schneeweißen deutlich krystallinischen Niederschlag. Die wässerige Lösung von Queksilberoxydsulfat, sowie die mit 5\% iger Schwefelsäure bereitete erzeugen nur eine Trübung: einen deutlichen Niederschlag, wie ich ihn mit denselben Reagenzien sogar in viel schwächeren Carnosinlösungen erhielt, habe ich nicht beobachtet. Sublimat ist zum Fällen des Methylguanidins ganz ungeeignet. 2,5\% ige PwS fällt das Methylguanidinnitrat noch aus einer Lösung der Substanz 1:6000, 25\% ige PwS erzeugt einen Niederschlag selbst in einer Lösung der Substanz 1:9000.

Die Resultate meiner Versuche sind in den 3 untenstehenden Tabellen zusammengestellt. Die 1. Tabelle enthält die Angaben über das freie Kreatinin, die 2. über das freie Carnosin, die 3. über das Methylguanidinnitrat. Selbstverständlich haben die angeführten Zahlen nur eine annähernde Bedeutung, da nicht nur die Herstellungsart der Reagenzien, sondern auch die Technik des Fällens selbst auf die Fällbarkeit einen Einfluß ausübt.

1) Diese Zeitschrift, Bd. 5, S. 72. 
Zur Kenntnis der Extraktivstoffe der Muskeln. XIII.

Tabelle 1.

Freies Kreatinin.

\begin{tabular}{|c|c|c|c|c|c|}
\hline Reagens & $\begin{array}{c}\text { Aussehen des } \\
\text { Niederschlags } \\
\text { und } \\
\text { Bedingungen, } \\
\text { unter denen er } \\
\text { sich bildet }\end{array}$ & $\begin{array}{l}\text { Mikroskopische } \\
\text { Eigenschaften } \\
\text { des } \\
\text { Niederschlags }\end{array}$ & $\begin{array}{l}\text { Konzen } \\
\text { grad in } \\
\text { welch } \\
\text { Trübu } \\
\text { ein Nie } \\
\text { noch } \\
\text { erhal- } \\
\text { ten } \\
\text { wird }\end{array}$ & $\begin{array}{l}\text { trations- } \\
\% \text {, bei } \\
\text { m eine } \\
\text { g oder } \\
\text { lerschlag } \\
\text { nicht } \\
\text { mehr } \\
\text { erhalten } \\
\text { wird }\end{array}$ & $\begin{array}{l}\text { Zeitraum, } \\
\text { in } \\
\text { welchem } \\
\text { der } \\
\text { Nieder- } \\
\text { schlag } \\
\text { oder } \\
\text { Trübung } \\
\text { entsteht }\end{array}$ \\
\hline $\mathrm{HgCl}_{2}$ & $\begin{array}{l}\text { weiß, amorph; } \\
\text { löst sich nicht } \\
\text { in einem großen } \\
\text { Überschuß des } \\
\text { Reagenten; } \\
\text { beim Stehen all- } \\
\text { mählicher Über- } \\
\text { gang in den kry- } \\
\text { stallinischen } \\
\text { Zustand }\end{array}$ & $\begin{array}{c}\text { gut ausgebildete } \\
\text { Prismen, stellen- } \\
\text { weise in hübsche } \\
\text { sternförmige } \\
\text { Drusen geordnet. } \\
\text { Feine, zu Kugeln } \\
\text { vereinigte } \\
\text { Nädelchen } \\
(1: 3000)\end{array}$ & $1 / 30$ & $1 / 40$ & $\begin{array}{c}1 / 20 \text { nach } \\
1 \text { Std. } \\
1 / 25-1 / 30 \\
\text { nach } \\
-5 \text { Std. }\end{array}$ \\
\hline $\begin{array}{c}\mathrm{Hg}\left(\mathrm{C}_{2} \mathrm{H}_{3} \mathrm{O}_{2}\right)_{2} \\
\mathrm{HgSO}_{4} \\
\mathrm{HgSO}_{4} \text { in } \\
5^{0} / 0 \mathrm{H}_{2} \mathrm{SO}_{4} \\
\mathrm{Hg}\left(\mathrm{NO}_{3}\right)_{2}\end{array}$ & \multicolumn{2}{|c|}{, } & - & $\begin{array}{l}1 \\
1 \\
1 \\
1\end{array}$ & - \\
\hline $\begin{array}{l}2,5 \% \\
\text { PWS }\end{array}$ & $\begin{array}{c}\text { weiß, amorph } \\
\text { bis } 1: 1000 \text {; bei } \\
\text { weiterer Ver- } \\
\text { dünnung kry- } \\
\text { stallinisch }\end{array}$ & $\begin{array}{c}\text { kurze, dicke, } \\
\text { polyedrische } \\
\text { Prismen; in einer } \\
\text { geringen Anzahl } \\
\text { rhomboidale } \\
\text { Tafeln mit abge- } \\
\text { stumpften } \\
\text { Winkeln }\end{array}$ & $1 / 280$ & $1 / 300$ & $\begin{array}{l}1 / 200 \text { nach } \\
5-6 \text { Std. } \\
1 / 280 \text { nach } \\
24 \text { Std. }\end{array}$ \\
\hline $\begin{array}{l}25 \% \\
\text { PWS }\end{array}$ & $\begin{array}{c}\text { amorph bis } \\
1: 3000 \text {, bei } \\
\text { größerer Ver- } \\
\text { dünnung krystal- } \\
\text { linisch }\end{array}$ & $\begin{array}{c}\text { Krystalle wie im } \\
\text { vorhergehenden } \\
\text { Fall }\end{array}$ & $1 / 850$ & $1 / 300$ & $\begin{array}{l}1 / 200 \text { nach } \\
5-6 \text { Std. } \\
1 / 250 \text { nach } \\
-24 \text { Std: }\end{array}$ \\
\hline
\end{tabular}


Tabelle 2.

Freies Carnosin.

\begin{tabular}{|c|c|c|c|c|c|}
\hline \multirow[t]{2}{*}{ Reagens } & \multirow{2}{*}{$\begin{array}{c}\text { Aussehen des Niederschlags } \\
\text { und Bedingungen, unter } \\
\text { denen er sich bildet }\end{array}$} & \multirow{2}{*}{\begin{tabular}{|c|} 
Mikro- \\
skopische \\
Eigen- \\
schaften \\
des \\
Nieder- \\
schlags
\end{tabular}} & \multicolumn{2}{|c|}{\begin{tabular}{|l|} 
Konzentrations- \\
grad in $\%$, bei \\
welchem eine \\
Trübung oder \\
ein Niederschlag
\end{tabular}} & \multirow{2}{*}{\begin{tabular}{|c} 
Zeitraum, \\
in \\
welchem \\
der \\
Nieder- \\
schlag \\
oder \\
Trübung \\
entsteht
\end{tabular}} \\
\hline & & & $\begin{array}{l}\text { noch } \\
\text { erhal- } \\
\text { ten } \\
\text { wird }\end{array}$ & $\begin{array}{c}\text { nicht } \\
\text { mehr } \\
\text { erhalten } \\
\text { wird }\end{array}$ & \\
\hline $\mathrm{HgCl}_{8}$ & $\left|\begin{array}{c}\text { weiß, flockig, locker, amorph; } \\
\text { in einem großen Überschuß des } \\
\text { Reagenten etwas löslich }\end{array}\right|$ & amorph & $1 / 20$ & $1 / 30$ & $\begin{array}{c}\text { 1/20 über } \\
\text { Nacht }\end{array}$ \\
\hline $\mathrm{g}\left(\mathrm{C}_{2} \mathrm{H}_{8} \mathrm{O}_{2}\right)_{2}$ & \multicolumn{2}{|l|}{ kein Niederschlag } & - & - & - \\
\hline $\mathrm{HgSO}_{4}$ & \begin{tabular}{|} 
weiß, locker, flockig, amorph. \\
Ein Ubberschuß des Reagenten \\
fördert das Ausfallen. Eingroßer \\
Überschuß löst den Nieder- \\
schlag nicht; vollständige Auf- \\
lösung in der Schwefelsäure
\end{tabular} & amorph & $1 / 1000$ & $1 / 2000$ & $\begin{array}{l}1 / 200-1 / 300 \\
\text { nach } \\
4-5 \text { Std. } \\
1 / 750-1 / / 000 \\
\text { nach } \\
10 \mathrm{Std}\end{array}$ \\
\hline $\begin{array}{l}\mathrm{HgSO}_{4} \\
\text { in } 5 \% \\
\mathrm{H}_{2} \mathrm{SO}_{4}\end{array}$ & $\begin{array}{l}\text { weiß, flockig, locker, amorph. } \\
\text { In einem großen Überschuß des } \\
\text { Reagenten löst er sich voll- } \\
\text { stăndig auf. Bei großer Ver- } \\
\text { dünnung }(1: 30000) \text { gallertartig }\end{array}$ & amorph & $1 / 1000$ & $\left.1 / 2000^{1}\right)$ & $\begin{array}{l}1 / 200-1 / 300 \\
\text { nach } \\
2-3 \text { Std. } \\
1 / 750-1 / 1000 \\
\text { nach } \\
5 \text { Std. }\end{array}$ \\
\hline $\mathrm{Hg}\left(\mathrm{NO}_{3}\right)_{2}$ & $\begin{array}{l}\text { gallertartig, weiß, amorph. Ein } \\
\text { großer Uberschuß des Rea- } \\
\text { genten löst den Niederschlag } \\
\text { nicht, sondern fördert das Aus- } \\
\text { fallen. Bei großer Verdünnung } \\
(1: 13500) \text { locker, flockig }\end{array}$ & amorph & $1 / 1000$ & $1 / 2000$ & $\begin{array}{l}1 / 195 \text { nach } \\
2 \text { Std. } \\
1 / 200 \text { nach } \\
4 \text { Std. } \\
1 / 800-1 / 1000 \\
\text { nach } \\
10 \mathrm{Std} .\end{array}$ \\
\hline $\begin{array}{l}2,5 \% \\
\text { PWS }\end{array}$ & $\begin{array}{c}\text { weiß, beim Stehen krystal- } \\
\text { linisch; bildet sich langsam; } \\
\text { löst sich nicht im Überschuß } \\
\text { des Reagenten }\end{array}$ & \begin{tabular}{|} 
kleine, \\
zu Kugeln, \\
Garben, \\
Bürsten \\
vereinigte \\
Naddelchen
\end{tabular} & 1/80 & $1 / 180$ & $\begin{array}{l}\text { 1/90 nach } \\
24 \text { Std. }\end{array}$ \\
\hline $\begin{array}{l}25 \% \\
\text { PWS }\end{array}$ & $\left|\begin{array}{c}\text { ebenso wie bei der Einwirkung } \\
\text { von 2,5\% PWS; bildet sich } \\
\text { rasch. Bei großer Verdä́nunung } \\
\text { körnig }(1: 20000)\end{array}\right|$ & $\begin{array}{c}\text { dasselbe } \\
\text { wie mit } \\
2,5 \% \\
\text { PWS }\end{array}$ & $1 / 200$ & 1/300 & $\begin{array}{l}\text { 1/186 nach } \\
5 \text { Std. } \\
1 / 200 \text { nach } \\
10 \text { Std. }\end{array}$ \\
\hline
\end{tabular}

1) Es fällt das gelbe pulverförmige basische Quecksilbersulfat aus. 
Zur Kenntnis der Extraktivstoffe der Muskeln. XIII.

Tabelle 3.

Methylguanidinnitrat.

\begin{tabular}{|c|c|c|c|c|c|}
\hline Reagens & $\begin{array}{c}\text { Aussehen des } \\
\text { Niederschlages und } \\
\text { Bedingungen, unter } \\
\text { denen er sich bildet }\end{array}$ & $\begin{array}{c}\text { Mikro- } \\
\text { skopische } \\
\text { Eigenschaften } \\
\text { des Nieder- } \\
\text { schlages }\end{array}$ & $\begin{array}{l}\text { Konzen } \\
\text { grad in } \\
\text { welch } \\
\text { Trübu } \\
\text { ein Nie } \\
\text { noch } \\
\text { erhal- } \\
\text { ten } \\
\text { wird }\end{array}$ & $\begin{array}{c}\text { trations- } \\
\% \text {, bei } \\
\text { m eine } \\
\text { ag oder } \\
\text { derschlag } \\
\text { nicht } \\
\text { mehr } \\
\text { erhalten } \\
\text { wird }\end{array}$ & $\begin{array}{c}\text { Zeitraum, } \\
\text { in } \\
\text { welchem } \\
\text { der } \\
\text { Nieder- } \\
\text { schlag } \\
\text { oder } \\
\text { Trübung } \\
\text { entsteht }\end{array}$ \\
\hline $\mathrm{HgCl}_{2}$ & kein Niedersc & chlag & - & 5 & - \\
\hline $\mathrm{Hg}\left(\mathrm{C}_{2} \mathrm{H}_{3} \mathrm{O}_{2}\right)_{2}$ & $\begin{array}{l}\text { schneeweiß, krystal- } \\
\text { linisch. In einem } \\
\text { großen Überschuß } \\
\text { des Reagenten etwas } \\
\text { löslich }\end{array}$ & $\begin{array}{l}\text { Nadeln, Keile, } \\
\text { Prismen } \\
\text { sargdeckel- } \\
\text { förmig }\end{array}$ & 3 & 2 & $\begin{array}{l}5 \text { nach } \\
2 \text { Std: } \\
3 \text { über } \\
\text { Nacht }\end{array}$ \\
\hline $\mathrm{HgSO}_{4}$ & $\begin{array}{l}\text { Trübung; in einem } \\
\text { Überschuß des } \\
\text { Reagenten unlöslich }\end{array}$ & $\begin{array}{c}\text { keine } \\
\text { Krystalle }\end{array}$ & 1 & $1 / 2$ & - \\
\hline $\begin{array}{c}\mathrm{HgSO}_{4} \\
\text { in } \\
\mathrm{H}_{2} \mathrm{SO}_{4}\end{array}$ & $\begin{array}{l}\text { Trübung; ein Über- } \\
\text { schuß des Reagenten } \\
\text { löst sie merklich, } \\
\text { doch bleibt eine } \\
\text { leichte Trübung } \\
\text { immer zurück }\end{array}$ & $\begin{array}{c}\text { keine } \\
\text { Krystalle }\end{array}$ & $1 / 2$ & $\left.1 / 8^{1}\right)$ & $\begin{array}{l}1 / 2 \text { eine } \\
\text { Trübung } \\
\text { nach } \\
24 \text { Std. }\end{array}$ \\
\hline $\mathrm{Hg}\left(\mathrm{NO}_{3}\right)_{2}$ & $\begin{array}{l}\text { schneeweiß, } \\
\text { krystallinisch }\end{array}$ & $\begin{array}{c}\text { Prismen, } \\
\text { meißelförmig } \\
\text { geschnitten }\end{array}$ & 3 & 2 & $\begin{array}{l}5 \text { nach } \\
2 \text { Std. } \\
3 \text { nach } \\
48 \text { Std. }\end{array}$ \\
\hline $\begin{array}{l}2,5 \% \\
\text { PWS }\end{array}$ & $\begin{array}{c}\text { weiß, } \\
\text { feinkrystallinisch }\end{array}$ & $\begin{array}{c}\text { feine lange } \\
\text { Prismen und } \\
\text { rhomboidale } \\
\text { Tafeln }\end{array}$ & $1 / 80$ & $1 / 90$ & $\begin{aligned} & 1 / 80 \text { nach } \\
& 1 \text { Std. } \\
& 1 / 60 \text { nach } \\
& 12 \text { Std. } \\
&\end{aligned}$ \\
\hline $\begin{array}{l}25 \% \\
\text { PWS }\end{array}$ & \multicolumn{2}{|c|}{$\begin{array}{l}\text { ein ebensolcher Niederschlag } \\
\text { wie im vorhergehenden Fall }\end{array}$} & $1 / 90$ & $1 / 100$ & $\begin{array}{l}1 / 80 \text { nach } \\
1 \text { Std. } \\
1 / 90 \text { nach } \\
12 \text { Std. }\end{array}$ \\
\hline
\end{tabular}

1) Bei großer Verdünnung fällt das gelbe basische Quecksilbersulfat als feines Pulver aus. 